\title{
ILQ Optimal Voltage Control for Biomass Free-Piston Stirling Engine Generator System
}

\author{
Ka Ahmadou*+, Massahiro Fujiwara**, Yusuke Nakamura***, Kazuki Sato**, Hiroshi Takami** \\ *Global course of Engineering and science Shibaura Institute of Technology 3-7-5 Toyosu, Koto-ku City, Tokyo 135-8548, \\ Japan, \\ **Shibaura Institute of Technology, Faculty of engineering, and Departmentt of Electrical Engineering Shibaura Institute of \\ Technology, 3 -7-5 Toyosu, Koto-ku Tokyo 135-8548, Japan \\ ***Department of Electrical Engineering and Computer science Shibaura Institute of Technology, 3-7-5 Toyosu, Koto-ku, Tokyo \\ 135-8548, Japan
}

(kaahmadou61@gmail.com, ae16073@shibaura-it.ac.jp, ma19064@shibaura-it.ac.jp, ae16033@shibaura-it.ac.jp, takami@shibaura-it.ac.jp)

+ Global course of Engineering and science Shibaura Institute of Technology 3-7-5 Toyosu, Koto-ku City, Tokyo 135-8548, Japan, kaahmadou61@gmail.com

Received: 20.01.2020 Accepted:22.03.2020

\begin{abstract}
In this paper, we study the control of output voltage of Free-Piston Stirling Engine Generator (SEG) System. The Stirling is normally used by connected to the grid. The grid is very robust because grid can be considered as infinite bus. When the SEG is applied to the based power-station in the remote rural or disaster areas, the SEG must be operated as a standalone system or off grid power system, this situation may lead to unstable generation. To solve the problem, the ILQ (Inverse Linear Quadratic) can be used to realize robust voltage controller. In this paper a new power generation system based on renewable energy sources is proposed specifically, wood pellet as the heat source. Indeed, the condition of the stirling engine is based on the voltage $V o$. The experimental results show that up to $1.6 \mathrm{~kW}$ can be generated from the generator system, and possible production of around $200 \mathrm{~L}$ of hot water $\left(45^{\circ} \mathrm{C}\right)$. In order to rescue us from serious conditions.
\end{abstract}

Keywords-Free-Piston Stirling Engine; Biomass; wood Pellet; ILQ optimal control;

\section{INTRODUCTION}

This The extensive use of fossil energies and their scarcity is impacting negatively in our global economy. Beside their scarcity, fossil fuels are the main cause of our environmental issues and the most common is "Global warming". This phenomenon is caused by the emission of several harmful gasses in the atmosphere. On the other hand, there are so many sources of renewable energy, easy to use and almost pollution-free, all over the world. For instance, biomass which is environmentally friendly and highly available.

In 1816, the Scottish reverend Robert Stirling invented an external combustion engine. From its inventor's name, the Stirling engine is also known as an internal heat engine which can run with any heat source. In other words, heat can be provided either from fossil fuels or clean energy sources such as solar, biomass and so forth. Moreover, air, steam, hydrogen or nitrogen are often utilized as working fluids. After this great invention, many types of Stirling engine have been developed over the years to improve its performance. However, starting from the 1962s, a new Stirling engine technology known as Free-Piston mechanical design. It has no crankshaft that makes its structure very simple, mechanically robust, with low friction loss the Free-Piston Stirling Engine (FPSE), was developed by a former Ohio University professor William Beale [1][2]. It was confirmed that the engine could have a long-life time, safety and high reliability [3]. Later, in Beale's company, FPSE was improved into a Free-Piston Stirling Engine Generator (FPSEG). This type of Stirling engine contains an integrated linear alternator to convert the mechanical energy into electrical power. Through the years, several applications have been developed using the FPSE such as space power system and many other features [4]. From burning biomass, specifically, the wood-pellet, an FPSEG was also used to produce alternative current up to $1 \mathrm{~kW}$ of $240 \mathrm{~V}, 50 \mathrm{~Hz}$ [5]. Using single or ganged free-piston Stirling engine, potential for generating biomass power has been studied in [6].

However, in this work, the FPSEG biomass system is used to produce electricity power using wood pellet as a heat source in the optimal operational condition.

\section{STATE EQUATION AND INVERSE REFERENCE MODEL OF SINGLE PHASE INVER}

\section{A. State equations of single phase inverter}

The voltage equation in the single-phase inverter circuit of Fig. 1 , is given by the following equations.

$$
v_{u v}=L_{F} \frac{d i_{u}}{d t}+r i_{u}+v_{o}
$$


K. Ahmadou et al., Vol.4, No.1, March, 2020

$$
i_{u}=C_{F} \frac{d v_{o}}{d t}+\frac{v_{o}}{R}+i_{o}
$$

Where, the terminal pair P-N $\mathrm{N}^{d t}$ the $\mathrm{DC}$ input terminal of the inverter, the terminal pair $\mathrm{u}-\mathrm{v}$ is an $\mathrm{AC}$ output terminal of the inverter. The terminal pair a-b is output terminal to the load, is the DC supply voltage, is the DC input current, and are the voltage and current of the output terminal of the inverter and, are the voltage and current of the output (load), and are the inductance, winding resistance and capacitance of the LC low pass filter (LPF) for the inverter output voltage and the current. is the output resistance.

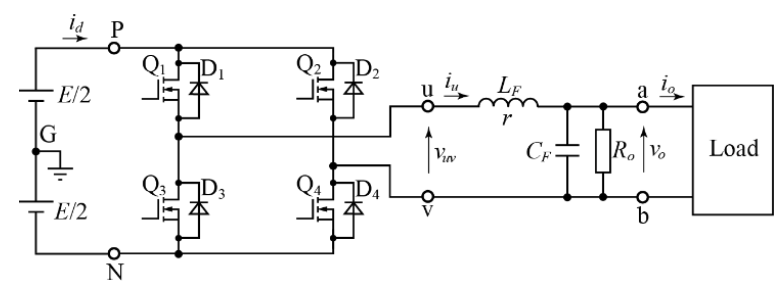

Fig. 1. Single-phase Inverter Circuit.

The state equation and output equation of the the plant at the time $t$ are given by the following equations, when the state variable is $x=\left[\begin{array}{ll}v_{o} & i_{u}\end{array}\right]^{\mathrm{T}}$.

$$
\left([]^{\mathrm{T}}: \text { transpose }\right)
$$

The input is $u=v_{u v}$, the disturbance is $d=i_{o}$ and the output is. $y=v_{o}$

$$
\begin{gathered}
\dot{x}=A x+B u+D d \\
y=C x
\end{gathered}
$$

When we define $T_{C}=R_{o} C_{F}$ and $T_{L}=L_{F} / r$ as time constants of the output circuit, the coefficient matrices in (3) and (4) are given by the following equations:

$$
A=\left[\begin{array}{cc}
-1 / T_{C} & 1 / C_{F} \\
-1 / L_{F} & -1 / T_{L}
\end{array}\right], B=\left[\begin{array}{c}
0 \\
1 / L_{F}
\end{array}\right], D=\left[\begin{array}{c}
-1 / C_{F} \\
0
\end{array}\right], C=\left[\begin{array}{ll}
1 & 0
\end{array}\right]
$$

Equations (3) and (4) satisfy the following conditions:

(i) Controllable and observable.

(ii) There is no zeros at the origin.

(iii) It is a minimum phase system.

(iv) Asymptotically stable without unstable poles.

From the above conditions (ii) and (iii), the zeros of the plant become the poles of the inverse model, then (3) and (4) can give a stable inverse model. At time $t$, subscript 0 is added to all variables of the inverter circuit in the ideal state with no error in the inverter parameters, and the following equation is obtained

$$
\begin{gathered}
\alpha+\beta \dot{x_{0}} t \cdot A x_{0}+\not\left(\mathbf{u}_{0}\right)+D d(1) \\
y_{0}=C x_{0}
\end{gathered}
$$

If the measured load current can be set as a disturbance in (5),

if the delay of the current sensor is ignored.

In (3) to (6), the deviation between the state variable and control input and output.

$$
\left.\begin{array}{l}
\Delta x=x-x_{0} \\
\Delta u=u-u_{0} \\
\Delta y=y-y_{0}
\end{array}\right\}
$$

Then the following error system is obtained

$$
\Delta \dot{x}=A \Delta x+B \Delta u
$$

$$
\Delta y=C \Delta x
$$

\section{B. Inverse reference model for voltage control}

An inverse reference model (IRM) that calculates reversely input and state variables to achieve ideal output, is derived from the state (5) and (6) in the ideal state. For the first line of (5), if the differential operator is set to $s$ and is obtained, the following equation is obtained.

$$
i_{u 0}=\frac{1+s T_{C}}{R_{o}} v_{o 0}+i_{o}
$$

Then, for the second line of Eq. (5), we obtain (11).

$$
v_{u v 0}=r\left(1+s T_{L}\right) i_{u 0}+v_{o 0}
$$

Equations (10) and (11) are not proper, then a first-order lag filter with time constant $T_{F}$ having the same order as the numerator is added. Therefore, the following inverse reference model is obtained as the reference input $y^{*}=v_{o 0}=v_{o}^{*}$ and disturbance $d=i_{0}$

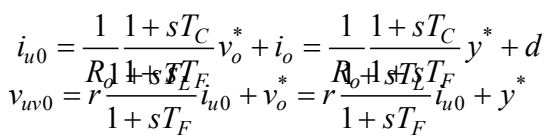

\section{ILQ optimal control response based on the proposed IRM}

1) $L Q$ problem and solution in servo system of error model In error model (8) and (9), in order to converge $\Delta x$ to zero and

eliminate the steady deviation of its target "0", a type-1 servo system appending one intergrator to the optimal regulator is shown in Fig. 2. Where $K_{F}, K_{I}$ are the state feedback gain and integral gain. 


\section{Optimal Servo Controller}

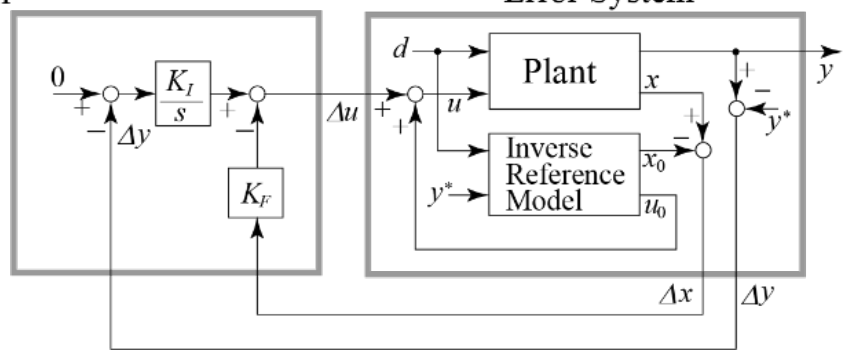

Fig. 2. Configuration of the type-1 servo system for error model based on inverse reference model.

Since the optimal servo controller in Fig. 2 has the same configuration as PI control only in the case of one input and one output, the optimal control of multivariable control can be positioned as a servo system developed from PI control to multidimensional feedback control.

Next, in order to replace the optimal servo design problem to the LQ problem, a new state variable is defined, and the servo system in Fig. 2 is equivalently converted to a closed loop system using only state feedback.

$$
\Delta z=z-z_{0}=\frac{\Delta y}{s}
$$

Where $z_{0}$ is the initial value of the integration of $\Delta y$.

An equivalent transformed closed-loop system is called an extended system because it has a structure that includes a controller in the state feedback. If the state variables $x_{e}=\left[\begin{array}{ll}\Delta x^{\mathrm{T}} & \Delta z^{\mathrm{T}}\end{array}\right]^{\mathrm{T}}$ and input $u_{e}=\Delta u$ of the extended system are defined the following equation is obtained from (8), (9), and (14).

$$
\dot{x}_{e}=A_{e} x_{e}+B_{e} u_{e}
$$

The coefficient matrices are given by the following equation.

$$
A_{e}=\left[\begin{array}{ll}
A & 0 \\
C & 0
\end{array}\right] \quad B_{e}=\left[\begin{array}{c}
B \\
0
\end{array}\right]
$$

From Fig. 2 and (14), following state feedback gain is obtained.

$$
u_{e}=-K_{e} x_{e} \quad K_{e}=\left[\begin{array}{ll}
K_{F} & K_{I}
\end{array}\right]
$$

As a result, the equivalent equation was transformed into a closedloop system in which state feedback (16) was applied to the state equation (15) of the expanded system. The LQ problem is given as a problem for finding a gain that minimizes the evaluation function given in quadratic form in a closed loop system. The evaluation function for the expanded state equation of (15) is given by the following equation using a positive definite weight matrix

$$
\begin{aligned}
& Q=C_{e}^{\mathrm{T}} C_{e}>0, R=R^{\mathrm{T}}>0 \\
& J_{e}=\int_{0}^{\infty}\left(x_{e}^{\mathrm{T}} Q x_{e}+u_{e}^{\mathrm{T}} R u_{e}\right) d t
\end{aligned}
$$

Where, $C_{e}$ is a weighting matrix in which the pair $\left(C_{e}, A_{e}\right)$ is detectable.

The problem of stabilizing the closed-loop system and minimizing the evaluation function (17) is called the SLQ problem. The solution $P_{0}$ of the following gain and optimal state feedback gain (Riccati equation).

$$
P_{0} A_{e}+A_{e}^{\mathrm{T}} P_{0}-P_{0} B_{e} R^{-1} B_{e}^{\mathrm{T}} P_{0}+Q=0
$$

By solving (18) the optimal state feedback gain is given by the following equation.

$$
K_{e}=R^{-1} B_{e}^{\mathrm{T}} P_{0}
$$

When pair $\left(C_{e}, A_{e}\right)$ is observable, the optimal solution $P_{0}$ becomes positive.

\section{2) From the $L Q$ problem to the ILQ design method}

The constant matrices $Q$ and $R$ in. (17) are weights for making a trade-off between the response of the state variable and the control input. Generally, it is determined by trial and error by repeating simulations and experiments. In contrast, the ILQ design method, which is an inverse approach to the LQ problem leads to an optimal solution by a simple pole assignment problem that gives a response specification of a closed-loop system. The solution can be obtained analytically with good visibility. Therefore, an optimal solution that can be adapted to changes in plant parameters can be obtained in a short time. In a plant that satisfies the above condition (i) to (iii), using the non-singular matrix $V$ and positive definite diagonal matrix $\Sigma$ and $K_{e}=V^{-1} \Sigma V\left[\begin{array}{ll}K_{F}^{0} & K_{I}^{0}\end{array}\right]$ according to the ILQ design method procedure. The ILQ optimal solution of (15) and (16) is given by:

$$
\left[\begin{array}{ll}
K_{F}^{0} & K_{I}^{0}
\end{array}\right]=\left[\begin{array}{ll}
K & I
\end{array}\right]\left[\begin{array}{ll}
A & B \\
C & 0
\end{array}\right]^{-1}
$$

When, $K_{F}^{0}$ and $K_{I}^{0}$ are defined reference optimal gains then $K=K_{F}^{0} A+K_{I}^{0} C, A_{K}=A-B K$ and $\Sigma$ is gain adjustment parameter.

3) Procedure for Deriving Reference Optimal Gain a) We obtain the relative degree of the $i$-th row of the transfer function matrix of the plant and confirm that the following decoupling matrix is non-singular. 
$D_{e}=\left[\begin{array}{c}c_{1} A^{d_{1}-1} B \\ \vdots \\ c_{m} A^{d_{m}-1} B\end{array}\right]$, and $d_{i}:=\min \left(k \mid c_{i} A^{k-1} B \neq 0, i=1 \ldots m\right)$

Where $c_{i} \quad$ is the $i$-th row vector of the coefficient matrix $C$.

$b)$ we find the polynomial matrix derived from the coefficient matrices.

$$
N_{\phi}(A)=\left[\begin{array}{c}
c_{1} \phi_{1}(A) \\
\vdots \\
c_{m} \phi_{m}(A)
\end{array}\right]
$$

Where $\phi_{i}(s)=s^{d_{i}}+\alpha_{i 1} s^{d_{i}-1}+\alpha_{i 2} s^{d_{i}-2}+\cdots+\alpha_{i d_{i}} \quad$ is a stable polynomial that determines the response specification of the closedloop system and $\alpha_{i 1}, \cdots, \alpha_{i d_{i}}$ are its coefficients.

c) The unknown gain of (20) that gives the optimal gain of the ILQ design method can be obtained from the following equation.

$$
K=D_{e}^{-1} N_{\phi}(A)
$$

In Fig. 2, when $\Sigma \rightarrow \infty$, the transfer function matrix (target transfer function) from the reference input to the output is given as a decoupling function with one-to-one correspondence between input and output.

$$
\begin{aligned}
& G_{y^{*} y}^{\infty}(s)= \operatorname{diag}\left\{\frac{\phi_{1}(0)}{\phi_{1}(s)}, \cdots, \frac{\phi_{m}(0)}{\phi_{m}(s)}\right\} \\
&=\operatorname{diag}\left\{\frac{\alpha_{11}}{s^{d_{1}}+\alpha_{1 d_{1}} s^{d_{1}-1}+\alpha_{1 d_{1}-1} s^{d_{1}-2}+\cdots+\alpha_{11}}, \cdots,\right. \\
& \\
&\left.\frac{\alpha_{m 1}}{s^{d_{m}}+\alpha_{m d_{m}} s^{d_{m}-1}+\alpha_{m d_{m}-1} s^{d_{m}-2}+\cdots+\alpha_{m 1}}\right\}
\end{aligned}
$$

On the other hand, in the ILQ servo system in order to minimize and optimize equation (17), the following three conditions should be satisfied.

$$
\begin{gathered}
\boldsymbol{E}=\boldsymbol{\Sigma}-\boldsymbol{K}_{\boldsymbol{V}} \boldsymbol{B}_{\boldsymbol{V}}-\left(\boldsymbol{K}_{\boldsymbol{V}} \boldsymbol{B}_{\boldsymbol{V}}\right)^{T}>0 \quad \boldsymbol{K}_{\boldsymbol{V}}:=V K \\
\boldsymbol{B}_{\boldsymbol{V}}:=B V^{-1} \\
\operatorname{Re} \lambda(\boldsymbol{F})=\operatorname{Re} \lambda\left(\boldsymbol{A}_{\boldsymbol{K}}+\boldsymbol{G H}\right)<0
\end{gathered}
$$

Where, $F=A_{K}+G H, \quad G=B_{V} E^{-1 / 2}, \quad H=E^{-1 / 2} K_{V} A_{K}$

$$
\left\|\boldsymbol{H}(s \boldsymbol{I}-\boldsymbol{F})^{-1} \boldsymbol{G}\right\|_{\infty}<1
$$

4) The analytical solution of ILQ optimal voltage control based on IRM of single-phase inverter.

The error models (8) and (9) satisfy the above condition. Therefore, the ILQ design method can be applied. When the reference optimal gain in Fig. 2 is obtained according to the solution procedure in previous section, it is given as an analytical solution as shown in (25) to (27).

Finally, we can obtain the ILQ analytique solution as:

Target transfer function:

$$
\begin{gathered}
G_{y^{*} y}^{\infty}(s)=\frac{\alpha_{11}}{s^{2}+\alpha_{12} s+\alpha_{11}} \\
K_{F}^{0}=\left[C_{F} L_{F}\left(\alpha_{12}-\frac{1}{T_{C}}\right) L_{F}\right]=\left[\begin{array}{ll}
K_{F 01} & K_{F 02}
\end{array}\right] \\
K_{I}^{0}=\left[C_{F} L_{F} \alpha_{11}\right]=\left[K_{I 01}\right]
\end{gathered}
$$

\section{SyStem COMPOSITION}

This system is based on the FPSEG using biomass as heat operation of the FPSEG consists of combustion using biomass, and cooling with water. The wood-pellets combustor provides heat to FPSEG's head. Meanwhile, the other part of the FPSEG is cooled by water. The heat swirls the FPSEG's head to warm the working helium gas. A temperature difference inside the FPSEG is induced by simultaneity from the cooling water. In fact, after starting up the FPSEG, the process of repeated heating and cooling of the FPSEG allows generating electricity. In fact, this cycle this repeated 25 times per second generating alternative current at $50 \mathrm{~Hz}$ as shown in Table I and Table II.

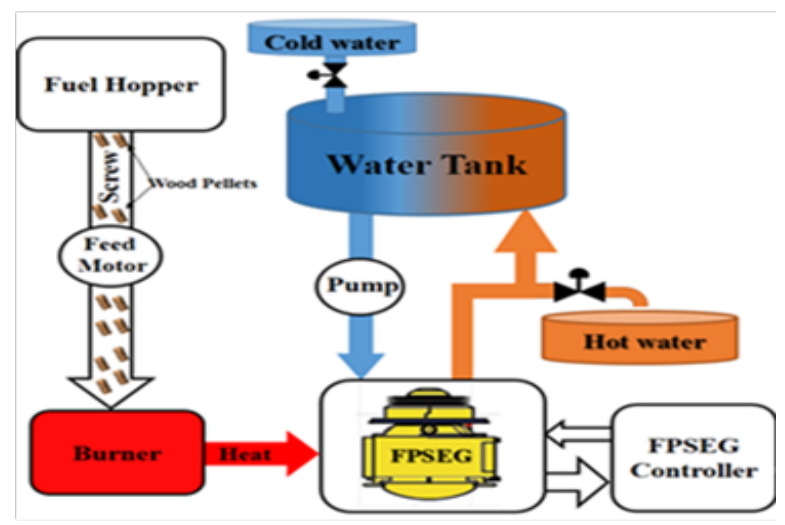

Fig. 3. Heat process system schematic

Table I. Specification of the FPSEG

\begin{tabular}{l|c}
\hline \multicolumn{2}{c}{ Free-Piston Stirling Engine (Microgen Engine } \\
\multicolumn{2}{c}{ Corporation) } \\
\hline Rated power & $1 \mathrm{~kW}$ \\
Rated voltage & $230 \mathrm{~V}$ \\
Operating frequency & $50 \mathrm{~Hz}$ \\
Weight & $50 \mathrm{Kg}$ \\
\hline
\end{tabular}


Table II. Parameters of the single-phase inverter

\begin{tabular}{ll}
\hline$L_{F}$ & $1 \mathrm{mH}$ \\
$r_{\mathrm{o}}$ & $13 \mathrm{~m} \Omega$ \\
$C_{F}$ & $11 \mu \mathrm{F}$ \\
$R_{o}$ & $10 \mathrm{k} \Omega$ \\
\hline
\end{tabular}

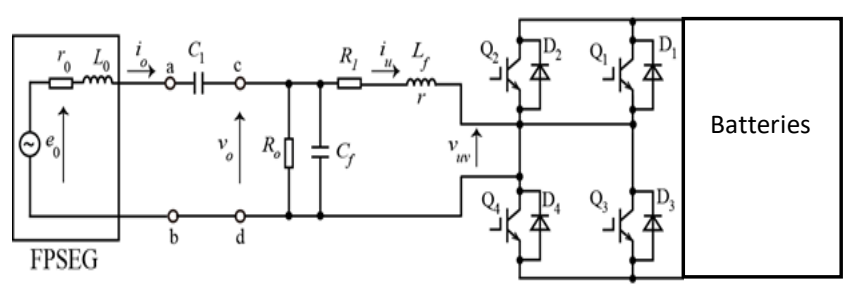

Fig. 4. Single-phase Inverter Circuit combine with FPSEG.

In Fig.4, the FPSEG is generating an AC voltage and the voltage Vo is set base on the biomass heating. However, this voltage can be control by voltage sensor. The terminal pair a-b is an AC output terminal of the FPSEG and $\mathrm{c}-\mathrm{d}$ is an DC input terminal of the converter. $i_{o}$ is the $\mathrm{AC}$ output current, $v_{u v}$ and $i_{u}$ are the voltage and current of the input terminal of the

converter, $v_{O}$ and $i_{O}$ are the voltage and current of the output of the FPSEG. In the other hand, $L_{F}$, ro, $C_{F}$, are the inductance, winding resistance and capacitance of the LC low pass filter (LPF) for smoothing the converter input voltage and the current, $R_{o}$ is the output resistance.

\section{Simulation and discussion}

In order to realize the stable production of electric power from the FPSEG, voltage command of inverter output passing through LC low pass filter is given as following sinusoidal waveform:

$$
v_{o}^{*}=v_{o 0}=\sqrt{2} V_{e} \sin \omega t
$$

Where $V_{e}$ and $\omega=200 \pi[\mathrm{rad} / \mathrm{s}]$ are the effective value and angular frequency of the inverter output voltage, respectively. In order to evaluate the robustness of the proposed voltage control, is changed at $170 \mathrm{~V}, 225 \mathrm{~V}$ and $170 \mathrm{~V}$ with transient process. On the other hand, angular frequency is kept constant. Table III shows the condition for simulated verification and the voltage $\mathrm{V}=170 \mathrm{~V}$ is more stable because it suppresses the temperature and get the maximum power by using the inverter.

Table III. Condition for Numerical Simulation.

\begin{tabular}{|c|c|c|}
\hline Time [s] & Mode Number & $V_{\boldsymbol{e}}[\boldsymbol{V}]$ \\
\hline $0.02 \sim 0.04$ & Mode 1 & 170 \\
\hline $0.04 \sim 0.065$ & Mode 2 & 255 \\
\hdashline $0.065 \sim 0.1$ & Mode 3 & 170 \\
\hline
\end{tabular}

Fig. 5 shows a result of numerical simulation. All symbols are referred to Fig. 4. Inverter output voltage $v_{o}$ is followed up to its command $v_{o}^{*}=v_{o 0}$ in any condition, minimizing velocity error between $v_{o}$ and $v_{o 0}$, due to proposed type- 1 servo system are reduced by applying an error model, based on the inverse reference model.

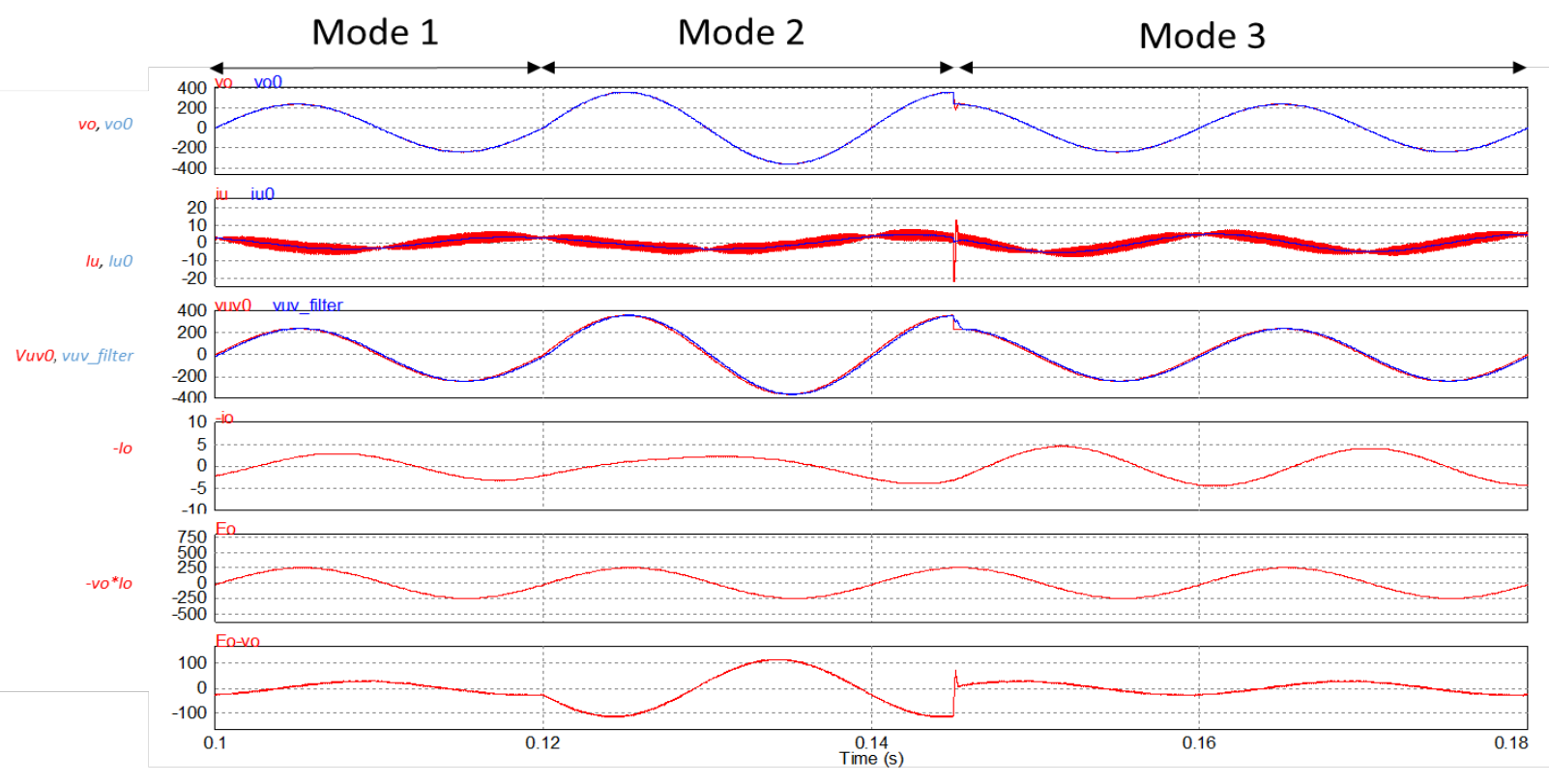

Fig. 5. Simulation result for single phase inverter with FPSE 


\section{CONCLUSION}

In this work we combine ILQ optimal controller with FPSEG used to generate power from biomass heat. The optimal and stable feedback gains are analytically determined by the ILQ designed method. Moreover, velocity errors due to type-1 servo system are reduced by applying an error model, based on the inverse reference model derived from voltage equation, to the ILQ servo-system. It is successfully obtained stable sinusoidal waveforms and excellent transient response to reference voltage command, suppressing distortion in the various loads with any power factor. Furthermore, this power generation system suitable for rural electrification disaster. Moreover, it can be used to supply the area where the national power grid has been damaged by eventual disaster.

\section{ACKNOWLEDGMENT}

I wish to thank Shibaura Institute of Technology, JICE and JICA for their support.

\section{REFERENCES}

[1] Akira Hoshi, lchinoseki, Shinsuke Sato and Kazuo Maezawa : "Investigation of Waste Heat Recovcry and Gcncratio Snystem by Free Piston Stirlin Egngine", The Japan Society of Mechanical Engineers, pp.269-272 (2010-6) (in Japanese).

[2] Hiroshi Takami, Takao Suzuki, Tomohiro Asaji, Sachio Furuki, Seirin Saito and Taro Hoshino: "Fundamental Electrical Characteristics and Maximum Power Operation of Free Piston Type Stirling Engine Generator on the Constant Heating Source", The IEE Japan, 5-59, pp. 345-350 (2015-8) (in Japanese)

[3] N. Ghanbaril, H. Golzari, H. Mokhtari, M. Poshtan, "Optimum location for operation of small size distributed generators", IEEE 6th International Conference on Renewable Energy Research and Application - ICRERA 2017, pp. 300-303, November 2017.
[4] Nahid-ur-RahmanChowdhury, Syed Enam Reza, TofaeelAhamedNitol, "Present Scenario of Renewable Energy in Bangladesh and a Proposed Hybrid System to Minimize Power Crisis in Remote

[5] Areas", INTERNATIONAL JOURNAL of RENEWABLE ENERGY RESEARCH,Vol.2, No.2, pp289-288,2012

[6] Abd-Al-Fattah-IbneMahabubJack G Sadly: "Overview of Free-Piston Stirling Engine Technology for Space Power Application”, Solar Energy Conference cosponsored by ASME, JSES, Honolulu, Hawaii, March 2227,1987

[7] M. Yesilbudak, M. Colak, R. Bayindir, "A review of data mining and solar power prediction", IEEE International Conference on Renewable Energy Research and Applications (ICRERA), pp. 1117-1121, 2016.

[8] Youcef BOT, A. Allali, "Using DGUPFC to Control the Voltage of MultiFeeder Smart Distribution System", International Journal of Renewable Energy Research-IJRER 2017, vol. 7, no. 4.

[9] M. R. Rashel, A. Albino, M. Tlemcani, T. C. F. Gonçalves, J. Rifath, "MATLAB simulink modeling of photovoltaic cells for understanding shadow effect", IEEE International Conference on Renewable Energy Research and Applications (ICRERA), pp. 747-750, 2016.

[10] J. Gary Wood Neill Lane: “Advanced 35kW Free-Piston Stirling Engine for Space Power Applications", Space Technology and Applications International Forum-STAIF, 2003

[11] W.-P. Schill, C. Gerbaulet, "Power system impacts of electric vehicles in Germany: Charging with coal or renewables?", Appl. Energy, vol. 156, pp. 185-196, Oct. 2015.

[12] Takami, and Suzuki: "Fundamental Electrical Characteristics and Maximum Power condition of the Free-Piston Stirling Engine Generator with series resonance circuit", Trans.IEE, Japan Vol.136-D, No.8, pp. 557-566,2016. (in Japanese)

[13] L. Schirone, M. Macellari, "A techno-economical analysis of charge management in electrical vehicles", 2015 Int. Canf Renew. Energy Res. Appl. ICRERA 2015, pp. 1253-1258, 2016.

[14] N. Liu, Q. Chen, X. Lu, J. Liu, J. Zhang, "A Charging Strategy for PVBased Battery Switch Stations Considering Service Availability and Self-Consumption ofm PV Energy", IEEE Trans. Ind. Electron., vol. 62, no. 8, pp. 4878-4889, Aug. 2015. 